\title{
STAR-CENTER POINTS OF UNIVALENT FUNCTIONS
}

\author{
NICOLAS K. ARTÉMIADIS
}

(Received 19 February 1990; revised 13 July 1990)

\begin{abstract}
Let $\mathscr{S}$ be the class of normalized univalent functions in the unit disk. For $f \in \mathscr{S}$ let $S_{f}$ be the set of all star center points of $f$. Let $\mathscr{S}_{0}=\left\{f \in \mathscr{S}: 0 \in S_{f}^{0}\right\}$ where $S_{f}^{0}$ is the interior of $S_{f}$. The influence that the size of the set $S_{f}^{0}$ has on the Taylor coefficients of a function $f \in \mathscr{S}_{0}$ is examined, and estimates of these coefficients depending only on $S_{f}^{0}$, as well as other results, are obtained.
\end{abstract}

1980 Mathematics subject classification (Amer. Math. Soc.) (1985 Revision): 30 C 50.

\section{Introduction}

Let $\mathscr{S}$ be the class of functions $f(z)=z+\sum_{n=2}^{\infty} a_{n} z^{n}$ which are analytic and univalent in the unit disk $D=\{z \in \mathbb{C}:|z|<1\}$. For $f \in \mathscr{S}$ the set $f(D)$ is a nonempty open connected proper subset of the complex plane $\mathbb{C}$. A point $w \in f(D)$ is called a star center point (s.c.p) of $f(D)$ if and only if

$$
t f(z)+(1-t) w \in f(D), \quad z \in D, 0 \leq t \leq 1 .
$$

For $f \in \mathscr{S}$, let $S_{f}$ be the set of all s.c.p of $f(D)$. Also let $\mathscr{S}_{0}$ be the subclass of $\mathscr{S}$ having the property that if $f \in \mathscr{S}_{0}$ then $0 \in S_{f}^{0}$, where $S_{f}^{0}$ is the interior of $S_{f}$.

In this paper we examine the influence that roughly the size of $S_{f}^{0}$ has on the Taylor coefficients, $a_{n}$, of a function in $\mathscr{S}_{0}$.

In Theorem 1, we obtain estimates of $\left|a_{n}\right|$, depending on the size of $S_{f}^{0}$ for $f \in \mathscr{S}_{0}$.

(C) 1991 Australian Mathematical Society 0263-6115/91 \$A2.00+0.00 
Theorem 2 provides additional information concerning the coefficient estimates obtained in Theorem 1. More precisely it is shown that if $f_{1}, f_{2} \in \mathscr{S}_{0}$ and $S_{f_{1}}^{0} \subset S_{f_{2}}^{0}$ then $B\left(f_{2}, n\right) \leq B\left(f_{1}, n\right), n=1,2, \ldots$, where $B\left(f_{1}, n\right)$, $B\left(f_{2}, n\right)$ are the estimates for the $n$th coefficients of $f_{1}$ and $f_{2}$ respectively. Finally we give examples of functions in $\mathscr{S}_{0}$ and compare our results with those obtained in [1].

I would like to thank the referees for their helpful comments on the subject.

\section{Preliminaries}

In this section we prove three lemmas which will be used later.

LEMMA 1. The set of all star center points of a function in $\mathscr{S}$ is convex.

Proof. Let $g \in \mathscr{S}, z_{1}, z_{2} \in D$ such that $g\left(z_{1}\right), g\left(z_{2}\right)$ belong to $S_{g}$. We show that the segment $\left[g\left(z_{1}\right), g\left(z_{2}\right)\right]$ is contained in $S_{g}$. Suppose $\left[g\left(z_{1}\right), g\left(z_{2}\right)\right] \not \subset S_{g}$ and let $w \in\left(g\left(z_{1}\right), g\left(z_{2}\right)\right)$ be such that $w \notin S_{g}$. Since $g\left(z_{1}\right), g\left(z_{2}\right)$ are s.c.p of $g(D)$ we have $w \in g(D)$.

By the hypothesis on $w$ there is $z_{0} \in D$ such that $\left[g\left(z_{0}\right), w\right] \not \subset g(D)$. Observe that if the points $g\left(z_{0}\right), g\left(z_{1}\right), g\left(z_{2}\right)$ are collinear then there is nothing to prove. Otherwise there is $w_{1} \in\left(g\left(z_{0}\right), w\right)$ such that $w_{1} \notin g(D)$. We have $\left[g\left(z_{1}\right), g\left(z_{0}\right)\right] \subset g(D)$ because $g\left(z_{1}\right) \in S_{g}$ and $g\left(z_{0}\right) \in g(D)$. Let $w_{2}$ be the intersection of the segment $\left[g\left(z_{1}\right), g\left(z_{0}\right)\right]$ and the straight line determined by the points $g\left(z_{2}\right)$ and $w_{1}$. These two sets intersect because $w_{1}$ is an interior point of the triangle $\left\{g\left(z_{0}\right), g\left(z_{1}\right), g\left(z_{2}\right)\right\}$. We have $w_{2} \in$ $g(D)$. Since $g\left(z_{2}\right) \in S_{g}$ it follows that $w_{1} \in g(D)$ which contradicts $w_{1} \notin$ $g(D)$. Hence $S_{g}$ is convex.

LEMMA 2. Let $f \in \mathscr{S}_{0}, \xi: D \rightarrow S_{f}^{0}$ be a univalent analytic function such that $\xi(0)=0, \xi(D)=S_{f}^{0}$, and let $z_{0}, z_{1}$ be complex numbers such that $\left|z_{0}\right|<\left|z_{1}\right|=r<1$. Then the segment $\left[f\left(z_{1}\right), \xi\left(z_{0}\right)\right]$ is contained in $f\left(\bar{D}_{r}\right)$, where $\bar{D}_{r}=\{z:|z| \leq r\}$.

Proof. For $\xi\left(z_{0}\right)=0$ the lemma is known [2, page 220]. Let $\rho$ and $\theta$ be two real numbers such that $0<\rho<1,-\pi \leq \theta \leq \pi, \rho e^{i \theta} z_{1}=z_{0}$. Put $\Phi(z)=t f(z)+(1-t) \xi\left(\rho e^{i \theta} z\right), z \in D, 0 \leq t \leq 1$. Clearly $\Phi$ is analytic in $D, \Phi(0)=f(0)=0$, and for each $z$ the point $\xi\left(\rho e^{i \theta} z\right)$ is a s.c.p of $f(D)$. Hence $\Phi$ is subordinate to $f$, so $\Phi(z)=f(\varphi(z))$, where $\varphi$ is analytic in 
$D$, and $|\varphi(z)| \leq|z|$. We have

$$
\Phi\left(z_{1}\right)=t f\left(z_{1}\right)+(1-t) \xi\left(\rho e^{i \theta} z_{1}\right)=t f\left(z_{1}\right)+(1-t) \xi\left(z_{0}\right)=f\left(\varphi\left(z_{1}\right)\right)
$$

and $\left|\varphi\left(z_{1}\right) \leq\right| z_{1} \mid$. Hence $\Phi\left(z_{1}\right) \in f\left(\bar{D}_{r}\right)$.

LEMMA 3. Let $n>2$ be an integer. Given $1 / 2 \leq x \leq 1$ and integers $1 \leq p \leq q$, define

$$
F_{q, p}=(p-x)(p+1-x) \cdots(q-x) .
$$

Then

$$
\begin{array}{rl}
-n ! n+n F_{n, 2}(x)+2 & x\left[F_{n, 3}(x)+2 ! 2 F_{n, 4}(x)+\cdots\right. \\
\left.+(n-2) !(n-2) F_{n, n}(x)+(n-1) !(n-1)\right] \leq 0 .
\end{array}
$$

Proof. We proceed by induction on $n$. Observe that (1) holds for $n=3$. We assume that it holds for $n$ and we prove that it holds for $n+1$. It suffices to show that the left-hand side of (1) is nonincreasing in $n$, for each fixed $x \in[1 / 2,1]$, or equivalently

$$
\begin{aligned}
(n+1) & F_{n+1,2}(x)-n F_{n, 2}(x)+2 x n ! n+2 x(n-x) \\
& \cdot\left[F_{n, 3}(x)+2 ! 2 F_{n, 4}(x)+\cdots+(n-1) !(n-1)\right] \\
\leq & (n+1) !(n+1)-n ! n .
\end{aligned}
$$

Now by the induction hypothesis we have

$$
2 x\left[F_{n, 3}(x)+2 ! 2 F_{n, 4}(x)+\cdots+(n-1) !(n-1)\right] \leq n ! n-n F_{n, 2}(x) .
$$

Hence (2) will hold if the following holds:

$$
\begin{aligned}
& (n+1) F_{n+1,2}(x)-n F_{n, 2}(x)+2 x n ! n+(n-x)\left(n ! n-n F_{n, 2}(x)\right) \\
& \quad \leq(n+1) !(n+1)-n ! n .
\end{aligned}
$$

This is equivalent to

$$
F_{n+1,2}(x)+n ! n x-(n+1) ! \leq 0 .
$$

To prove (4) we proceed as follows. We put $\Phi(x)=F_{n+1,2}(x)+n ! n x$ $-(n+1)$ ! and we claim that the derivative $\Phi^{\prime}(x)$ is nonnegative for $1 / 2 \leq$ $x \leq 1$. If this is proven it will mean that $\Phi(x)$ is nondecreasing so that its maximum value will be taken for $x=1$. But since $\Phi(1)=0$ (4) will be proven.

We show that

$$
\Phi^{\prime}(x)=n ! n+F_{n+1,2}^{\prime}(x) \geq 0, \quad 1 / 2 \leq x \leq 1 .
$$


Observe that from the definition of $F_{q, p}(x)$ it follows that

$$
F_{n+1,2}^{\prime}(x)=-F_{n+1,2}(x) \cdot \sum_{k=2}^{n+1} \frac{1}{k-x},
$$

so (5) can be written

$$
n ! n-F_{n+1,2}(x) \cdot \sum_{k=2}^{n+1} \frac{1}{k-x} \geq 0 .
$$

Since $1 / 2 \leq x \leq 1$, to prove (7) it suffices to show

$$
n ! n-F_{n+1,2}\left(\frac{1}{2}\right) \cdot \sum_{k=2}^{n+1} \frac{1}{k-\frac{1}{2}} \geq 0 .
$$

We again proceed by induction on $n$. It is easily seen that (8) holds for $n=3$. Assume that it holds for $n$. To show that (8) holds for $n+1$ we prove that the left-hand side of $(8)$ is nondecreasing in $n$, or that

$$
(n+1) !(n+1)-n ! n \geq F_{n+1,2}\left(\frac{1}{2}\right)\left[\left(n+\frac{1}{2}\right) \cdot \sum_{k=2}^{n+1} \frac{1}{k-\frac{1}{2}}+1\right] \text {. }
$$

If in (9) the expression $F_{n+1,2}\left(\frac{1}{2}\right)$ is replaced by

$$
n ! n / \sum_{k=2}^{n+1} \frac{1}{k-\frac{1}{2}}
$$

we get

$$
\begin{aligned}
(n+1) !(n+1)-n ! n \geq & {\left[\frac{n ! n}{\sum_{k=2}^{n+1}} \frac{1}{k-\frac{1}{2}}\right] } \\
& \cdot\left[\left(n+\frac{1}{2}\right) \cdot \sum_{k=2}^{n+1} \frac{1}{k-\frac{1}{2}}+1\right] .
\end{aligned}
$$

Since (8) holds, it follows that (9) is true if $(10)$ holds. But (10) is equivalent to

$$
\frac{n}{n+2} \leq \sum_{k=1}^{n} \frac{1}{2 k+1}, \quad n \geq 3,
$$

which is easily seen to be true by induction. It follows that (9) holds, and this proves the lemma.

\section{The main results}

We wish to give coefficient estimates for the Taylor expansion of a function in $\mathscr{S}_{0}$. 
Let $f \in \mathscr{S}_{0}$. From Lemma 1 it follows that $S_{f}^{0}$ is convex. Also $S_{f}^{0} \neq \mathbb{C}$ since $f(C) \neq \mathbb{C}$.

Let $\alpha$ be any point of $S_{f}^{0}$. Riemann's Mapping Theorem asserts that there is a unique analytic function

$$
g_{\alpha}: S_{f}^{0} \rightarrow D
$$

having the properties
(a) $g_{\alpha}(\alpha)=0$ and $g_{\alpha}^{\prime}(\alpha)>0$,
(b) $g_{\alpha}$ is one-to-one,
(c) $g_{\alpha}\left(S_{f}^{0}\right)=D$.

Put

$$
\mu(f, \alpha)=\left[1-\left|g_{\alpha}(0)\right|^{2}\right] / g_{\alpha}^{\prime}(0) .
$$

THEOREM 1. Let $f(z)=z+\sum_{n=2}^{\infty} a_{n} z^{n}$ be a function in $\mathscr{S}_{0}$ and let $\alpha$ be a point of $S_{f}^{0}$. Then

(i) $0<\mu(f, \alpha) \leq 1$.

(ii) If $\mu(f, \alpha)=1$ then $\left|a_{n}\right| \leq 1, n=1,2, \ldots$.

(iii) $\mu(f, \alpha)=1$ if and only if $S_{f}^{0}=f(D)$.

(iv) If $\mu(f, \alpha)<1$ then $\left|a_{n}\right| \leq A_{n}(f, \alpha)+R_{n-1}(\sigma)=M_{n}(f, \alpha), n \geq 2$, where $A_{n}(f, \alpha)=1+(n-1) \prod_{k=2}^{n}(k-1) /(k-\sigma), \sigma=1 /(1+\mu(f, \alpha))$, and

$$
\begin{aligned}
R_{n}(\sigma)=\frac{-n ! n}{\prod_{k=2}^{n+1}(k-\sigma)}+ & \frac{n}{n+1-\sigma}+\frac{2 \sigma}{n+1-\sigma} \\
\cdot & {\left[\frac{1}{2-\sigma}+\frac{2 ! 2}{(2-\sigma)(3-\sigma)}+\cdots\right.} \\
& \left.+\frac{(n-2) !(n-2)}{(2-\sigma) \cdots(n-1-\sigma)}+\frac{(n-1) !(n-1)}{(2-\sigma) \cdots(n-\sigma)}\right] .
\end{aligned}
$$

(v) $\left|a_{n}\right| \leq B(f, n), n \geq 2$, where $B(f, n)=\inf _{\alpha \in S_{f}^{0}}\left(M_{n}(f, \alpha)\right)$.

Proof. Put $g=g_{\alpha}^{-1}$ where $g_{\alpha}$ is the function defined in (12). Then $g: D \rightarrow S_{f}^{0}$ is analytic in $D$ and has the following properties:

(a') $g(0)=\alpha, g^{\prime}(0)=1 / g_{\alpha}^{\prime}(\alpha)>0$;

$\left(b^{\prime}\right) g$ is one-to-one;

(c') $g(D)=S_{f}^{0}$.

Let $g_{\alpha}(0)=\beta$. Then $\beta \in D$ and $g(\beta)=0$.

Put

$$
G(z)=g\left(\frac{z+\beta}{1+\bar{\beta} z}\right), \quad z \in D .
$$

The function $G: D \rightarrow S_{f}^{0}$ is analytic in $D$ and has the following properties: 
(a") $G(0)=g(\beta)=0 ; G^{\prime}(0)=g^{\prime}(\beta)\left(1-|\beta|^{2}\right)=\left(1-|\beta|^{2}\right) / g_{\alpha}^{\prime}(0)=$ $\left(1-\left|g_{\alpha}(0)\right|^{2}\right) / g_{\alpha}^{\prime}(0)$;

$\left(b^{\prime \prime}\right) G$ is one-to-one;

(c") $G(D)=S_{f}^{0}$.

Clearly $G$ is subordinate to $f$. It follows that

$$
G(z)=f(\omega(z))
$$

where $\omega$ is analytic on $D$ and $|\omega(z)| \leq|z|$.

Put $G(z)=\sum_{n=1}^{\infty} b_{n} z^{n}, z \in D$. We have since, $G^{\prime}(0)$ does not vanish, that

$$
0<b_{1}=G^{\prime}(0)=\omega^{\prime}(0)=\left[1-\left|g_{\alpha}(0)\right|^{2}\right] / g_{\alpha}^{\prime}(0)=\mu(f, \alpha) \leq 1 .
$$

This proves assertion (1) of Theorem 1 .

The function $G(z) / b_{1}=\sum_{n=1}^{\infty}\left(b_{n} / b_{1}\right) z^{n}$ belongs to the class $\mathscr{S}_{0}$ and maps $D$ onto the region $\left(1 / b_{1}\right) S_{f}^{0}=\left\{w / b_{1}: w \in S_{f}^{0}\right\}$ which is convex since $S_{f}^{0}$ is convex. It follows that

$$
\left|b_{n} / b_{1}\right| \leq 1, \quad n=1,2, \ldots
$$

Observe that $\omega$ is univalent in $D$ because the composition of two univalent functions is univalent.

Summarizing the properties of $\omega$, we have:

(i) $\omega$ is univalent in $D$;

(ii) $\omega(D) \subset D$;

(iii) $\omega(0)=0$;

(iv) $0<\omega^{\prime}(0)=b_{1} \leq 1$.

If in addition we had $\omega(D)=D$ then we would have $\omega(z)=z, \omega^{\prime}(0)=$ $b_{1}=1$ and it would follow from (14) and (16) that $G(z)=f(z)$ so that $a_{n}=b_{n},\left|a_{n}\right| \leq 1$. This proves assertion (ii) of Theorem 1 .

Next assume that $\omega(D)$ is a proper subset of $D$. Then it follows from the condition for equality in Schwarz's lemma that $\omega^{\prime}(0)<1$.

The above imply

(i) $\omega(D)=D$ if and only if $\omega^{\prime}(0)=1$,

(ii) if $\omega^{\prime}(0)<1$ then $0<b_{1}<1$,

(iii) $\left|b_{n}\right| \leq\left|b_{1}\right| \leq 1$,

and assertion (iii) of Theorem 1 follows from (17)(i).

Let $z, z_{0} \in D$ such that $\left|z_{0}\right|<|z|=r<1$. Put $G\left(z_{0}\right)=w \in S_{f}^{0}$, $f(z)-w=R e^{i \tau}, z=r e^{i \theta}$. It follows from Lemma 2 that $w$ is a s.c.p of $f\left(\bar{D}_{r}\right)$. Therefore

$$
\frac{\partial}{\partial \theta} \arg [f(z)-w]=\frac{\partial \tau}{\partial \theta} \geq 0
$$


We have

$$
\log [f(z)-w]=\log R+i \tau
$$

so

$$
\operatorname{Im}\left[\frac{\partial}{\partial \theta} \log (f(z)-w)\right] \geq 0 .
$$

In view of

$$
\frac{\partial}{\partial \theta}=i r e^{i \theta} \frac{d}{d z}=i z \frac{d}{d z}
$$

we get

$$
\operatorname{Re}\left[z f^{\prime}(z) /\left(f(z)-G\left(z_{0}\right)\right)\right] \geq 0 \text {. }
$$

The last inequality holds for all $z, z_{0}$ in $D$ for which $|z|>\left|z_{0}\right|$. Therefore if $\lambda$ is a real number such that $0 \leq \lambda<1$, we have

$$
\operatorname{Re}\left[z f^{\prime}(z) /(f(z)-G(-\lambda z))\right] \geq 0, \quad z \in D .
$$

Put

$$
F(z)=\left[z f^{\prime}(z) /(f(z)-G(-\lambda z))\right]=\sum_{n=0}^{\infty} c_{n} z^{n}, \quad z \in D .
$$

It is easily seen that $F$ is analytic in $D$ and that $c_{0}=1 /\left(1+b_{1} \lambda\right)$.

Due to the inequality

$$
\operatorname{Re} F(z) \geq 0, \quad z \in D
$$

we have

$$
\left|c_{n}\right| \leq 2 c_{0}=\frac{2}{1+b_{1} \lambda}
$$

From (18) we get

$$
z f^{\prime}(z)=\sum_{n=1}^{\infty} n a_{n} z^{n}=\sum_{n=1}^{\infty}\left[a_{n}-b_{n}(-\lambda)^{n}\right] z^{n} \cdot \sum_{n=0}^{\infty} c_{n} z^{n} .
$$

The last equation gives

$$
n a_{n}=\sum_{k=1}^{n}\left[a_{k}-(-\lambda)^{k} b_{k}\right] c_{n-k}, \quad n=1,2, \ldots,
$$

or

$$
\left(n-c_{0}\right) a_{n}=\sum_{k=1}^{n-1} a_{k} c_{n-k}-\sum_{k=1}^{n}(-\lambda)^{k} b_{k} c_{n-k} .
$$

If we set $\lambda=0$ then (20) and (19) provide the well known inequality $\left|a_{n}\right| \leq$ $n, n=2,3, \ldots$. 
From (20) we obtain, on account of (16) and (19),

$$
\begin{aligned}
\left|a_{n}\right| & \leq \frac{2 c_{0}}{n-c_{0}} \sum_{k=1}^{n-1}\left|a_{k}\right|+\frac{1}{n-c_{0}} \sum_{k=1}^{n} \lambda^{k}\left|c_{n-k} \| b_{k}\right| \\
& \leq \frac{2 c_{0}}{n-c_{0}} \sum_{k=1}^{n-1}\left|a_{k}\right|+\frac{\lambda^{n} b_{1} c_{0}}{n-c_{0}}+\frac{1}{n-c_{0}} \sum_{k=1}^{n-1} 2 b_{1} c_{0} \lambda^{k} .
\end{aligned}
$$

Now if we let $\lambda \rightarrow 1$ we get, since $b_{1} \sigma=1-\sigma$, that

$$
\left|a_{n}\right| \leq \frac{2 \sigma}{n-\sigma} \sum_{k=1}^{n-1}\left|a_{k}\right|+\frac{(1-\sigma)(2 n-1)}{n-\sigma}, \quad n \geq 2 .
$$

From (21) we deduce that for $n \geq 2$,

$$
\left|a_{n}\right| \leq A_{n}(f, \alpha)+R_{n-1}(\sigma)=M_{n}(f, \alpha) \leq A_{n}(f, \alpha) .
$$

The last part of (22) follows immediately from Lemma 3 , since $R_{n}(\sigma)$ is nonpositive for $n \geq 1$ and $1 / 2 \leq \sigma \leq 1$.

To prove the first part of (22) we proceed by induction on $n$. It is easily seen that for $n=2,3,(21)$ provides

$$
\begin{gathered}
\left|a_{2}\right| \leq 1+\frac{1}{2-\sigma}=A_{2}(f, \alpha)+R_{1}(\sigma)=A_{2}(f, \alpha), \\
\left|a_{3}\right| \leq 1+\frac{2 ! 2}{(2-\sigma)(3-\sigma)}=A_{3}(f, \alpha)+R_{2}(\sigma)=A_{3}(f, \alpha),
\end{gathered}
$$

because $R_{1}(\sigma)=R_{2}(\sigma)=0$, which proves that (22) holds for $n=2,3$. Assume that (22) holds for $n$. We get from (21), after some calculations, that

$$
\begin{aligned}
\left|a_{n+1}\right| & \leq \frac{2 \sigma}{n+1-\sigma} \sum_{k=1}^{n}\left|a_{k}\right|+\frac{(1-\sigma)(2 n+1)}{n+1-\sigma} \\
& \leq A_{n+1}(f, \alpha)+R_{n}(\sigma)=M_{n+1}(f, \alpha) .
\end{aligned}
$$

It follows that (22) holds for $n+1$. This proves assertion (iv) of Theorem 1 , while assertion (v) is obvious. The theorem is proved.

REMARK. If in (19) and (16) equality holds for $n=2,3,4$ then for $c_{1}=c_{2}=c_{3}=2 \sigma, b_{2}=b_{4}=-b_{1}, b_{3}=b_{1}, \lambda=1$, it is easily checked that (22) is sharp for $n \leq 4$. Indeed we find

$$
\begin{gathered}
a_{2}=1+\frac{1}{2-\sigma}, \quad a_{3}=1+\frac{4}{(2-\sigma)(3-\sigma)}, \\
a_{4}=1+\frac{18}{(2-\sigma)(3-\sigma)(4-\sigma)}+\frac{\sigma^{2}-\sigma}{(2-\sigma)(3-\sigma)(4-\sigma)} .
\end{gathered}
$$

However the sharpness of (22) for all $n$ remains open. 
We make the following conjecture.

Conjecture. Let $f \in \mathscr{S}_{0}, \alpha \in S_{f}^{0}$. Then

$$
\left|a_{n}\right| \leq A_{n}(f, \alpha)+R_{n-1}(\sigma)+H_{n}(\sigma), \quad n \geq 2,
$$

where

$$
H_{n}(\sigma)=\sum_{k=3}^{n-2}\left[R_{k}(\sigma)(2 \sigma)^{n-k-1} / \prod_{p=5}^{n+3-k}(p-\sigma)\right],
$$

for $n \geq 5$ and $H_{n}(\sigma)=0$ for $n<5$.

Furthermore, if equality holds in (16) and (19) and if

$$
c_{n}=2 \sigma, \quad b_{2 q}=-b_{1}, \quad b_{2 q-1}=b_{1}, \quad n=1,2, \ldots, q=1,2, \ldots,
$$

then for the $a_{n}$ obtained from (20), (*) is sharp.

ThEOREM 2. Let $f_{1}, f_{2}$ be functions in $\mathscr{S}_{0}$. Let $B\left(f_{1}, n\right), B\left(f_{2}, n\right)$ be the corresponding bounds on the Taylor coefficients of $f_{1}$ and $f_{2}$ respectively, as these are defined in Theorem 1(v). Suppose $S_{f_{1}}^{0} \subset S_{f_{2}}^{0}$. Then

$$
B\left(f_{2}, n\right) \leq B\left(f_{1}, n\right) .
$$

Proof. Let $\alpha \in S_{f_{1}}^{0}$. Let $G_{1}$ be the function obtained from $f_{1}$ exactly the same way as $G$ was obtained from $f$ in (13). Similarly, since $\alpha$ also belongs to $S_{f_{2}}^{0}$, let $G_{2}$ be the function obtained from $f_{2}$. We have

$$
G_{1}(D)=S_{f_{1}}^{0} \subset S_{f_{2}}^{0}=G_{2}(D), \quad G_{1}(0)=G_{2}(0)=0,
$$

and both $G_{1}$ and $G_{2}$ are regular and univalent in $D$. It follows that $G_{1}$ is subordinate to $G_{2}$, so $G_{1}(z)=G_{2}(\varphi(z))$, where $\varphi$ is analytic in $D$ and $|\varphi(z)| \leq|z|$. We have $G_{1}^{\prime}(z)=G_{2}^{\prime}(\varphi(z)) \varphi^{\prime}(z)$, or

$$
G_{1}^{\prime}(0)=\mu\left(f_{1}, \alpha\right)=G_{2}^{\prime}(0) \varphi^{\prime}(0)=\mu\left(f_{2}, \alpha\right) \varphi^{\prime}(0) .
$$

Since $\left|\varphi^{\prime}(0)\right| \leq 1$ we have

$$
\mu\left(f_{1}, \alpha\right) \leq \mu\left(f_{2}, \alpha\right)
$$

Put

$$
\sigma_{1}=\frac{1}{1+\mu\left(f_{1}, \alpha\right)}, \quad \sigma_{2}=\frac{1}{1+\mu\left(f_{2}, \alpha\right)} .
$$

We have from (24) that

$$
\sigma_{1} \geq \sigma_{2}
$$


Now the function $M_{n}(f, \alpha)=A_{n}(f, \alpha)+R_{n-1}(\sigma)$, defined in the statement of Theorem 1, can be written as follows

$$
\begin{aligned}
M_{n}(f, \alpha)=1 & +\frac{n-1}{n-\sigma}+\frac{2 \sigma}{n-\sigma} \\
& \cdot\left[\frac{1}{2-\sigma}+\frac{2 ! 2}{(2-\sigma)(3-\sigma)}+\cdots+\frac{(n-2) !(n-2)}{(2-\sigma) \cdots(n-1-\sigma)}\right] .
\end{aligned}
$$

It is easily seen that the derivative of $M_{n}(f, \alpha)$ with respect to $\sigma$ is nonnegative, which implies that $M_{n}(f, \alpha)$ is an increasing function of $\sigma$. It follows that

$$
M_{n}\left(f_{1}, \alpha\right) \geq M_{n}\left(f_{2}, \alpha\right) .
$$

By taking the infinum of the left side of (26) for $\alpha \in S_{f_{1}}^{0}$ and of the right side for $\alpha \in S_{f_{2}}^{0}$, we get (23) because $S_{f_{1}}^{0} \subset S_{f_{2}}^{0}$. This proves the theorem.

\section{Examples and comments}

EXAMPLe (from [1]). The function

$$
f(z)=\frac{1}{2 \varepsilon}\left[\left(\frac{1+z}{1-z}\right)^{\varepsilon}-1\right], \quad z \in D, 1<\varepsilon<2,
$$

belongs to the class $\mathscr{S}_{0}$. This is easily seen if we sketch $f(D)$. More precisely let $L_{1}, L_{2}$ be the rays which start from the point $(-1 / 2 \varepsilon, 0)$ and make with the positive $x$-axis the angles $(2-\varepsilon) \frac{\pi}{2},(\varepsilon-2) \frac{\pi}{2}$ respectively. Then $S_{f}^{0}$ is the open set which contains the origin and is bounded by the rays $L_{1}, L_{2}$. Let $T$ be the symmetric set of $S_{f}^{0}$ with respect to the line $x=-1 / 2 \varepsilon$. Then $f(D)=\mathbb{C}-\bar{T}$.

If we choose $\alpha=0 \in S_{f}^{0}$ then the function $G$ considered in (13), which maps $D$ onto $S_{f}^{0}$, is

$$
G(z)=\frac{1}{2 \varepsilon}\left[\left(\frac{1+z}{1-z}\right)^{2-\varepsilon}-1\right], \quad z \in D,
$$

and we have $\mu(f, 0)=G^{\prime}(0)=(2-\varepsilon) / \varepsilon$ and $\sigma=\varepsilon / 2$.

Other examples can be found in [2, pages 196, 197].

We close with the following comment.

In [1] the authors present a different approach to the subject. Given $f \in \mathscr{S}$ the index $\delta$ of starlikeness of $f$ is defined to be

$$
\delta=\sup \{r: f(z) \text { is a s.c.p of } f(D), \text { whenever }|z|<r\} .
$$


Let $\Delta_{\delta}$ be the class of all starlike functions whose index is equal to $\delta$, $0 \leq \delta \leq 1$. For $f \in \Delta_{\delta}$ the following inequality holds:

$$
\left|a_{n}\right| \leq \prod_{k=1}^{n-1} \frac{k(1+\delta)+1-(-\delta)^{k}}{k(1+\delta)+\delta+(-\delta)^{k}}
$$

The estimates given by (27) depend on $\delta$, or equivalently on the size of $f\left(D_{\delta}\right)$ which (in the cases of interest, that is, when $0<\delta<1$ ) is always a bounded subset of $S_{f}^{0}$.

On the other hand the estimates, given in Theorem 1 above, depend on the entire set $S_{f}^{0}$. If $S_{f}^{0}$ is unbounded (see example given above) then $f\left(D_{\delta}\right)$ is a proper subset of $S_{f}^{0}$. Now it is possible in this case (that is, when $S_{f}^{0}$ is unbounded) that the "unused" part of $S_{f}^{0}$ "hides" some additional information on the $a_{n}$, including some concerning the sharpness of (27).

\section{References}

[1] L. Raymon and D. E. Tepper, 'Star center points of starlike functions', J. Austral. Math. Soc. (Series A) 19 (1975).

[2] Z. Nehari, Conformal mapping, McGraw-Hill, New York, 1952.

\section{9, Megalou Alexandrou Street}

\section{Thrakomakedones}

Athens

Greece 